\title{
Non-canonical Wnt induces chondrocyte de-differentiation through Frizzled 6 and DVL-2/B-raf/CaMKIla/syndecan 4 axis
}

\author{
Zhe Xie $^{1} \cdot$ Mostafa Khair $^{1} \cdot$ Irfan Shaukat $^{1} \cdot$ Patrick Netter $^{1} \cdot$ Didier Mainard $^{1} \cdot$ Lydia Barré $^{1} \cdot$ Mohamed Ouzzine $^{1}$
}

Received: 5 July 2017 / Revised: 20 November 2017 / Accepted: 29 November 2017 / Published online: 19 January 2018

(c) ADMC Associazione Differenziamento e Morte Cellulare 2018

\begin{abstract}
Dysregulation of Wnt signaling has been implicated in developmental defects and in the pathogenesis of many diseases such as osteoarthritis; however, the underlying mechanisms are poorly understood. Here, we report that non-canonical Wnt signaling induced loss of chondrocyte phenotype through activation of Fz-6/DVL-2/SYND4/CaMKII $/$ B-raf/ERK1/2 cascade. We show that in response to Wnt-3a, Frizzled 6 (Fz-6) triggers the docking of CaMKII $\alpha$ to syndecan 4 (SYND4) and that of B-raf to DVL-2, leading to the phosphorylation of B-raf by CaMKII $\alpha$ and activation of extracellular signalregulated kinase 1 and 2 (ERK1/2) signaling, which leads to chondrocyte de-differentiation. We demonstrate that CaMKII $\alpha$ associates and phosphorylates B-raf in vitro and in vivo. Our study reveals the mechanism by which non-canonical Wnt activates ERK1/2 signaling that induces loss of chondrocyte phenotype, and demonstrates a direct functional relationship between CaMKII $\alpha$ and B-raf during chondrocyte de-differentiation. The identification of Fz-6, SYND4, and B-raf as novel physiological regulators of chondrocyte phenotype may provide new potential anti-osteoarthritic targets.
\end{abstract}

\section{Introduction}

Wnts signal through multiple pathways $[1,2]$, the best characterized of which is the $\mathrm{Wnt} / \beta$-catenin signaling, also known as the "canonical" pathway. Canonical Wnt signaling is initiated by binding of Wnt proteins to a Frizzled-LRP5/6 receptor complex [3]. This leads to activation of the protein Disheveled (Dsh/DVL) and inhibition of phosphorylation events that otherwise target $\beta$-catenin for proteasomal destruction, resulting in its stabilization in the cytoplasm. The stabilized $\beta$-catenin accumulates and translocates to the nucleus, where it interacts with transcription factors of the T-cell-specific transcription factor/lymphoid enhancerbinding factor family to activate the transcription of target genes [4]. In contrast to the above mechanism, Wnts can instead activate other signaling pathways referred to as

Edited by R.A. Knight.

Electronic supplementary material The online version of this article (https://doi.org/10.1038/s41418-017-0050-y) contains supplementary material, which is available to authorized users.

Mohamed Ouzzine

mohamed.ouzzine@univ-lorraine.fr

1 UMR7365 CNRS-University of Lorraine, Biopôle, Faculty of Medicine, 54505 Vandoeuvre-lès-Nancy, France "non-canonical", which does not involve stabilization of $\beta$ catenin such as the planar cell polarity pathway [5] and the $\mathrm{Ca}^{2+} / \mathrm{CaMKII}$ pathways [2, 6]. Recently, several studies connect Wnt signaling with bone disease, osteoarthritis (OA), and cancer [7-9]; therefore, understanding their complex signaling mechanisms and biological roles is of wide interest.

Besides Wnts, the syndecans (SYNDs) are a family of four (transmembrane) TM proteoglycans, which control cell proliferation, adhesion, and migration [10, 11]. Of mammalian SYND family members, SYND4 acts as co-receptors for growth factors and transduces signals via the intracellular domain, by interacting with numerous effectors [11].

In the cartilage, SYND4 seems to be induced specifically in hypertrophic chondrocytes and its expression is elevated in human OA cartilage $[12,13]$. In addition, SYND4-null mice were shown to develop less severe OA-like cartilage destruction [13].

In this study, we report that non-canonical Wnt-3a signaling induces loss of chondrocyte phenotype through activation of Fz-6/DVL-2/SYND4/CaMKII $\alpha /$ B-raf/ERK1/2 cascade. We show that SYND4 docks CaMKII $\alpha$ and DVL2 interacts with B-raf in a Wnt-dependent manner, leading to activation of B-raf by CaMKII $\alpha$ and to de-differentiation of chondrocytes. To our knowledge, this is the first report 
describing the partners and the interactions between them and other components of the non-canonical Wnt/ERK1/2 pathway and provides molecular insights for non-canonical Wnt-driven chondrocyte de-differentiation. It also reveals a physiological function of SYND4 and CaMKII $\alpha$ in this process.

\section{Results}

\section{Non-canonical Wnt/ERK1/2 signaling mediates chondrocyte de-differentiation}

Wnt- $3 \mathrm{a}$ is expressed in the cartilage and has an established role in OA [14]. We first investigated the effect of Wnt-3a on the expression of chondrocyte-lineage markers COL2A1, $S O X 9$, and aggrecan in human primary chondrocytes and articular cartilage explants. The expression of COL2A1, SOX9, and aggrecan was decreased by Wnt-3a in cartilage explants (Fig. 1a). We also found that the expression of the hypertrophy marker RUNX2 was increased (Fig. 1a). Similar results were obtained with human primary chondrocytes (data not shown). These results indicate that Wnt3a activates de-differentiation of articular chondrocytes. Accordingly, treatment of human primary chondrocytes with Wnt-3a produced a shift from round and polygonal cells to fibroblast-like cells (Fig. 1b). We next investigated whether Wnt-3a mediates loss of chondrocyte phenotype through canonical Wnt pathway. Knockdown of $\beta$-catenin failed to rescue the Wnt-3a down-regulation of COL2AI (Fig. 1c), indicating that Wnt-3a regulates COL2Al through $\beta$-catenin-independent pathway and suggests that downregulation of COL2A1 expression and loss of chondrocyte phenotype by Wnt-3a is mediated through non-canonical Wnt signaling pathway in human primary chondrocytes.

It has been shown that Wnt-3a also signals through $\beta$ catenin-independent mechanisms to mediate distinct physiologically relevant responses $[15,16]$. Wnt-3a activates extracellular signal-regulated kinase 1 and 2 (ERK1/2) signaling in primary chondrocytes. As shown in Fig. 1d, the phospho-ERK (p-ERK) level was increased in a timedependent manner starting within $5 \mathrm{~min}$ of the treatment with Wnt-3a and last over $2 \mathrm{~h}$. Co-treatment with the MEK inhibitor, U0126, abrogated the activation of ERK1/2 pathway by Wnt-3a (Fig. 1e). To determine whether noncanonical Wnt/ERK1/2 pathway mediates Wnt-3a-induced down-regulation of COL2Al, we analyzed the effect of inhibition of Wnt-3a-induced activation of ERK1/2 on COL2A1 expression. Interestingly, inhibition of ERK1/2 pathway rescued Wnt-3a-induced down-regulation of COL2Al (Fig. 1f), thus revealing that Wnt-3a inhibits the expression of COL2Al through activation of ERK1/2 pathway. Consistent with this finding, knockdown of ERK2
(Fig. 1g) reverted the effect of Wnt-3a on the expression of COL2A1 (Fig. 1h), confirming the key role of ERK1/2 in Wnt-3a-induced down-regulation of COL2Al and chondrocyte de-differentiation.

\section{DVL-2 docks B-raf to activate Wnt/ERK1/2 signaling}

To investigate the molecular mechanisms involved in the activation of ERK1/2 by Wnt-3a, we explored the role of DVLs. DVL is a key component of both canonical and noncanonical Wnt signaling pathways and, depending on the cellular context, specifically activates each Wnt signaling branch [17]. Mammalian DVLs include three isoforms, DVL-1, DVL-2, and DVL-3 [18-20]. Analysis of their expression by Western blotting showed that DVL-3 is the most abundant DVL expressed in human primary chondrocytes, whereas DVL-1 is the least abundant (Fig. 2a). In order to determine the role of DVLs in Wnt-3a-induced dedifferentiation of chondrocytes, we knocked down each of DVLs individually (Fig. 2b-d) and evaluated the effect on Wnt-3a-induced decrease in the expression of COL2A1 and activation of ERK1/2 signaling. DVL-2 knockdown not only prevented down-regulation of COL2Al by Wnt-3a but also promoted its expression (Fig. 2e). The knockdown of DVL-1 and DVL-3 did not prevent Wnt-3a-induced downregulation of COL2Al, instead silencing of DVL-1 potentiates it (Fig. 2e). These results indicate that DVL-2 mediates Wnt-3a down-regulation of COL2Al and suggests that, in chondrocytes, non-canonical Wnt pathway signal through DVL-2. Next, we asked whether DVL-2 silencing affects activation of ERK1/2 in response to Wnt-3a. Although Wnt-3a induces the activation of ERK1/2 signaling, it failed to stimulate this pathway in chondrocytes made deficient in DVL-2 (Fig. 2f). The knockdown of DVL-2 provoked a decline, rather than increase in ERK1/2 activation in response to Wnt-3a, indicating that DVL-2 is essential for the activation of ERK1/2 signaling by Wnt-3a.

DVL-2 is the scaffold protein that relays Wnt signaling by bridging receptor and adaptor proteins [21, 22]; therefore, we performed co-immunoprecipitation assays to analyze whether DVL-2 interacts with B-raf which transduces signals to MEK and ERK1/2 MAPK. Coimmunoprecipitation with anti-B-raf-specific antibodies showed that DVL-2 associates with B-raf and this occurs only in the presence of Wnt-3a (Fig. 3a); therefore, revealing that B-raf interacts with DVL-2 in Wnt-dependent manner. To determine whether recruitment of DVL-2 to the membrane is required for DVL-2 interaction with B-raf, we used a DVL-2 mutant lacking the DEP domain (Flag-DVL2 $\triangle 361-736)$ that is important for membrane recruitment [23]. We stimulated cells containing the Flag-tagged wildtype or $\Delta 361-736$ mutant of DVL-2 with Wnt-3a and then immunoprecipitated B-raf with specific antibodies. As 
Fig. 1 Wnt-3a induces loss of chondrocyte phenotype via a $\beta$-catenin-independent pathway. a Wnt-3a down-regulates the expression of COL2A1, aggrecan, and $S O X 9$ and up-regulates that of RUNX2 in human cartilage explants $(n=5)$, as evaluated by Q-PCR. Values were normalized for the housekeeping gene ribosomal protein S29 and are expressed as relative expression compared with control. b Primary human chondrocyte morphology under invert microscope $(\times 10)$. With Wnt-3a treatment, round and polygonal chondrocytes were shifted to fibroblast-like cells. c Fold changes of COL2A1 mRNA expression normalized to control in primary chondrocytes transfected with siRNA to $\beta$-catenin or siRNA control (Sicontrol) and treated with either Wnt-3a or vehicle $(n=3)$. d Detection of phosphorylated and total ERK1/2 in human primary chondrocytes treated with either Wnt-3a or vehicle for different time points. $\beta$-Actin was used as a loading control $(n=3)$. e Detection of phosphorylated and total ERK1/2 in human primary chondrocytes treated with Wnt-3a or vehicle, in the presence or absence of MEK1/2 inhibitor U0126. $\beta$-Actin was used as a loading control $(n=3)$. f Fold changes of COL2A1 mRNA expression normalized to control in primary chondrocytes pretreated with the MEK1/2 inhibitor U0126 and treated with either Wnt-3a or vehicle $(n=3)$. g Detection of MAPK1 and MAPK3 in primary chondrocytes transfected with siRNA specific to MAPK1 (Si-MAPK1), MAPK3

(Si-MAPK3), or siRNA control

(Si-control). $\beta$-Actin was used as a loading control $(n=3)$. h Fold changes of COL2A1 mRNA expression normalized to control in primary chondrocytes transfected with siRNA specific to MAPK1, MAPK3, or siRNA control and treated with either Wnt-3a or vehicle $(n=4)$. One representative blot of three independent experiments is shown. Data are expressed as mean \pm S.D. of separate experiments. Statistical analysis was performed with an unpaired Student's $t$ test $(* p<0.05$;

$* * p<0.01)$ $\mathbf{a}$

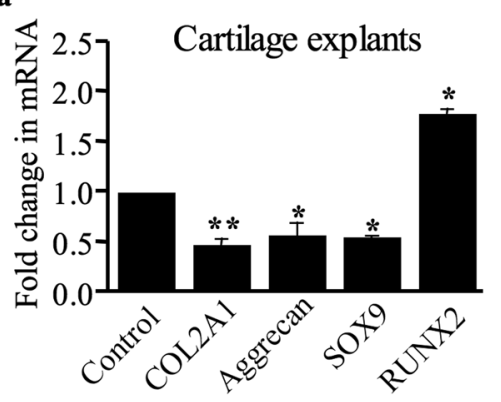

b

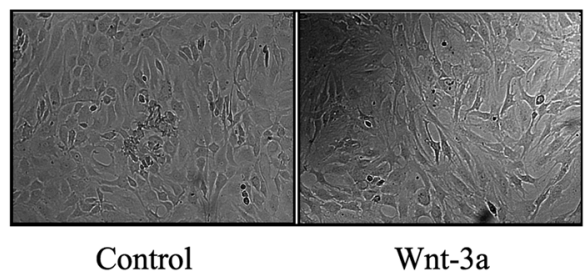

c

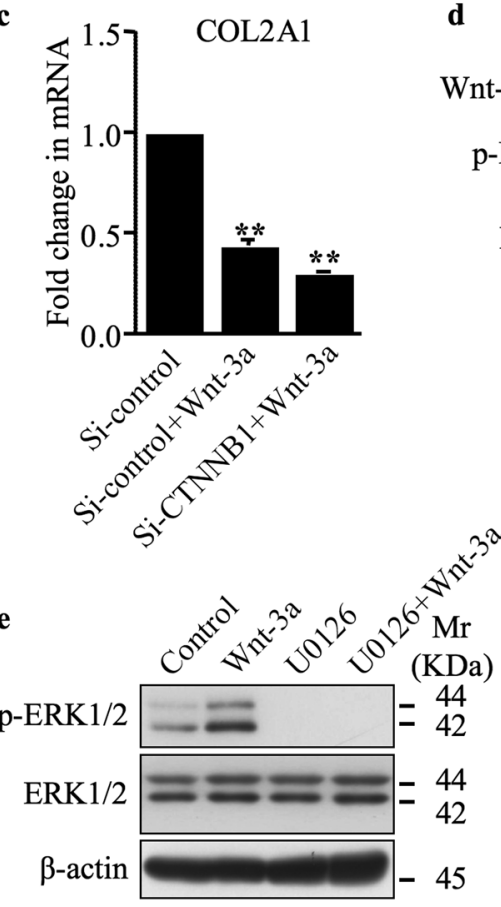

$\mathrm{Mr}$

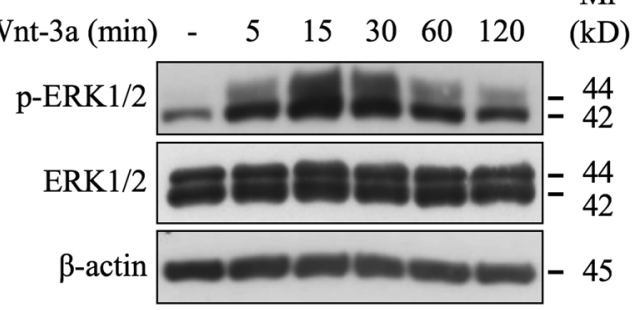

f

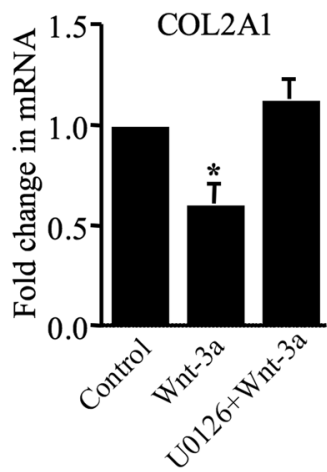

h

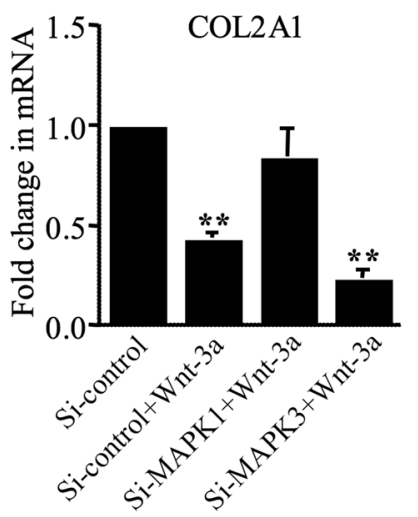


a

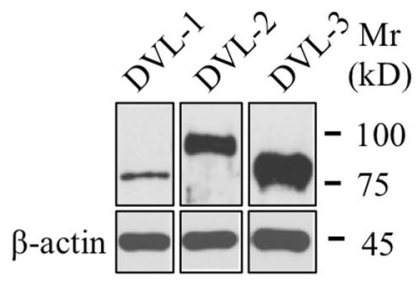

b

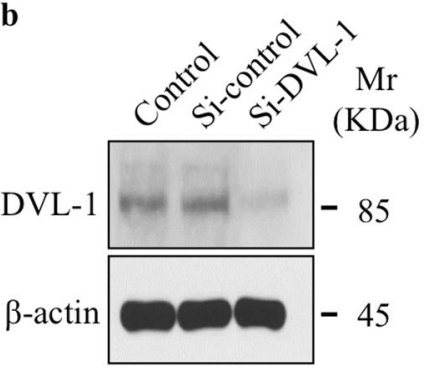

c

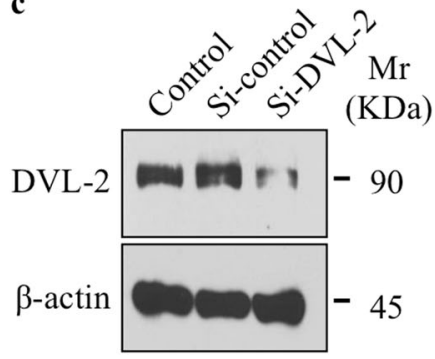

d

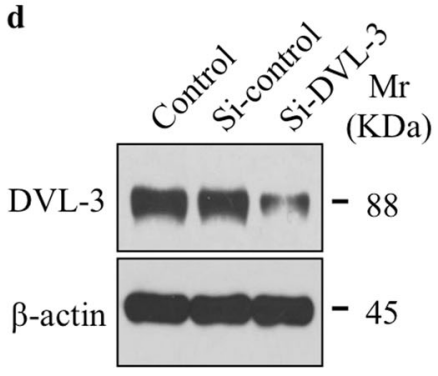

e

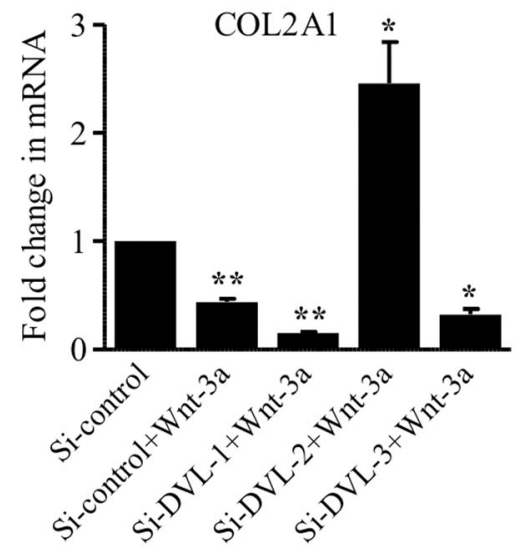

f

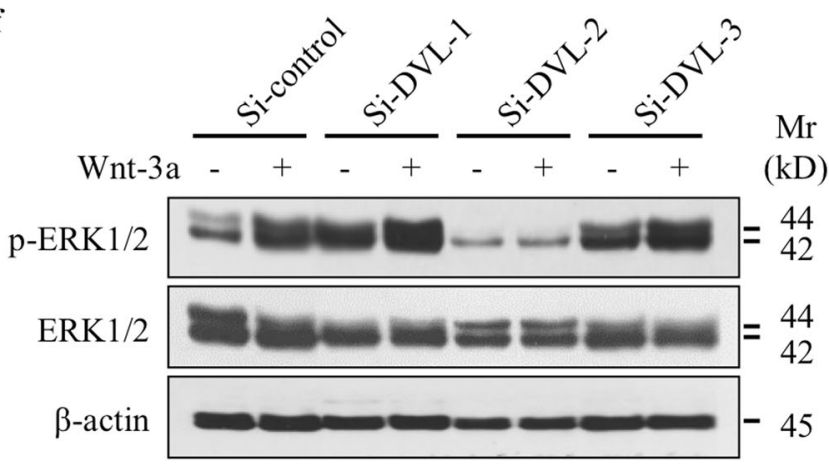

Fig. 2 DVL-2 mediates non-canonical Wnt-induced ERK1/2 activation and down-regulation of COL2A1 expression. a Detection of total DVL subtypes. $\beta$-actin was used as a loading control $(n=3)$. b Detection of DVL-1, c DVL-2 and d DVL-3 in human primary chondrocytes with or without transfection with siRNA specific to DVL-1 (Si-DVL-1), DVL-2 (Si-DVL-2), DVL-3 (Si-DVL-3), or siRNA control (Si-control). $\beta$-Actin was used as a loading control ( $n$ $=3$ ). e Fold changes of COL2A1 expression normalized to control in primary chondrocytes transfected with siRNA specific to DVL-1, DVL-2, DVL-3, or siRNA control and treated with Wnt-3a $(n=3)$.

shown in Fig. 3b, the Wnt-3a-dependent binding between DVL-2 and B-raf was observed only in the presence of Wnt-3a in cells expressing the wild-type DVL-2, but not in the DVL-2 $(\Delta 361-736)$ mutant. These results indicate that DVL-2 interaction with B-raf requires the presence of the DEP domain and suggest that recruitment of DVL-2 to the membrane is critical for DVL-2 docking of B-raf. Taken together, these data strongly suggest that activation of f Detection of phosphorylated and total ERK1/2 in primary chondrocytes transfected with siRNA specific to DVL-1, DVL-2, DVL-3, or siRNA control and treated or not with Wnt-3a or vehicle. $\beta$-Actin was used as a loading control $(n=3)$. Q-PCR values were normalized for the housekeeping gene ribosomal protein S29 and are expressed as the relative expression compared with control. One representative blot of three independent experiments is shown. Data are expressed as mean \pm S.D. Statistical analysis was performed with an unpaired Student's $t$ test $(* p<0.05 ; * * p<0.01)$

ERK1/2 pathway by Wnt-3a is regulated through recruitment of DVL-2 to the plasma membrane and docking of Braf. Next, we analyzed whether B-raf was activated by Wnt$3 \mathrm{a}$ and found that phosphorylation of B-raf was markedly increased in a time-dependent manner (Fig. 3c), indicating that Wnt-3a activates B-raf in chondrocytes. To investigate the role of B-raf in Wnt-3a-induced activation of ERK1/2 signaling pathway, we stimulated chondrocytes 
Fig. 3 Wnt-3a induces the docking of B-raf to DVL-2 and activates B-raf to transduce signal to ERK1/2. a Detection of DVL-2 and B-raf in cell lysates of primary chondrocytes treated with Wnt-3a or vehicle after immunoprecipitation with antiB-raf antibodies. Total cell lysates were analyzed by immunoblot with anti-DVL-2 and anti-B-raf antibodies. $\beta$ Actin was used as a loading control $(n=3)$. b Detection of Flag-tagged DVL-2 and B-raf proteins in cell lysates of primary chondrocytes, transfected with expression vector for Flag-tagged wild-type DVL-2 or Flag-DVL-2 $(\Delta 361-736)$ and treated with Wnt-3a or vehicle, after immunoprecipitation with antiB-raf antibodies. Total cell lysates were analyzed by immunoblot with anti-Flag and anti-B-raf antibodies. $\beta$-Actin was used as a loading control $(n=3)$. $\mathbf{c}$ Detection of phosphorylated and total B-raf in primary chondrocytes treated with Wnt-3a or vehicle for different time points. $\beta$-Actin was used as a loading control $(n=3)$. d Detection of phosphorylated and total ERK1/ 2 and B-raf in primary chondrocytes transfected with either empty vector or wild-type B-raf-expressing vector and treated with Wnt-3a or vehicle. $\beta$-Actin was used as a loading control $(n=3)$. e Detection of phosphorylated and total ERK1/ 2 and B-raf in primary chondrocytes transfected with either empty vector or with the vector expressing the dead mutant of B-raf (D594V) and treated with Wnt-3a or vehicle. $\beta$-Actin was used as a loading control $(n=3)$. One representative blot of three independent experiments is shown
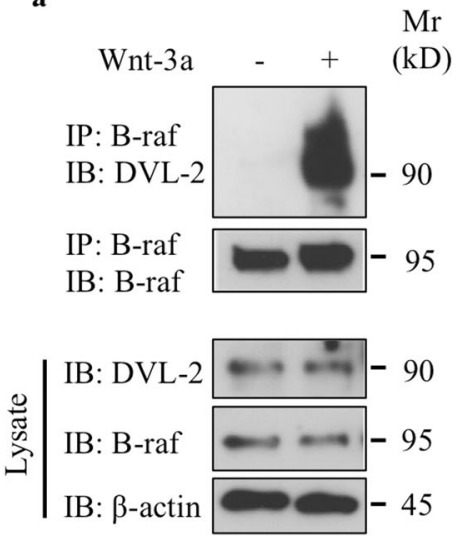

c

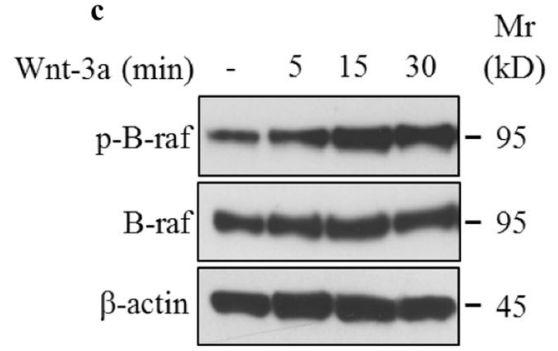

d

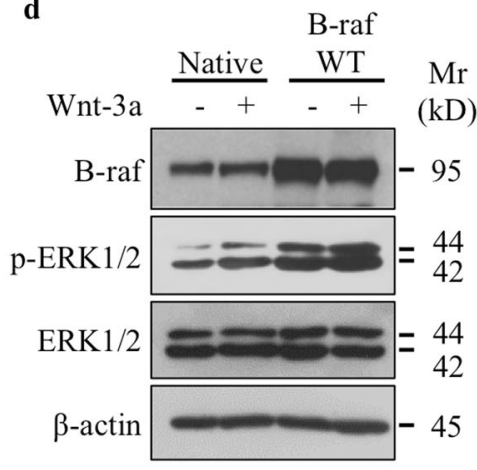

b

Flag-DVL-2(WT) $+-+\quad-\mathrm{Mr}$

Flag-DVL-2( $\Delta 361-736)+\quad+\quad+\quad(\mathrm{kD})$
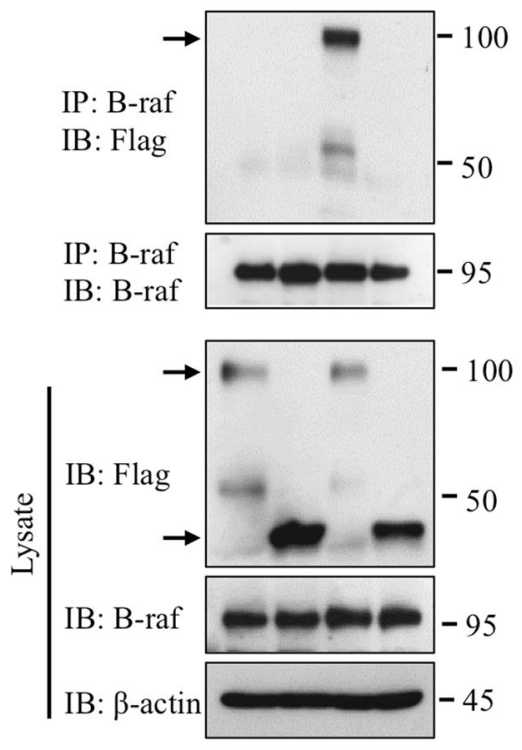

e

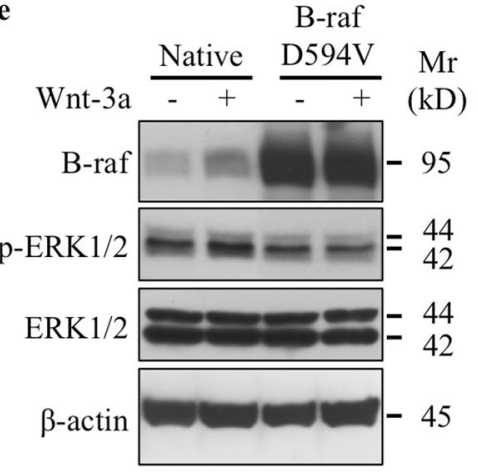

containing the wild-type or D594V kinase-dead mutant of B-raf with Wnt-3a and then analyzed the phosphorylation of ERK1/2. As shown in Fig. 3d, forced expression of wildtype B-raf increased the phosphorylation of ERK1/2, which was increased further in response to Wnt-3a. In contrast, expression of D594V mutant significantly reduced basal activation of ERK1/2 and prevented the activation of ERK1/2 by Wnt-3a (Fig. 3e), indicating that B-raf mediates Wnt-3a-induced activation of ERK1/2 signaling pathway.

\section{$\mathrm{Ca}^{2+} / \mathrm{CaMKII}$ mediates non-canonical Wnt activation of ERK1/2}

To determine whether Wnt-3a activates the non-canonical $\mathrm{Ca}^{2+} / \mathrm{CaMKII-dependent} \mathrm{pathway} \mathrm{in} \mathrm{chondrocytes,} \mathrm{we}$ 
Fig. 4 Wnt-3a activates CaMKII $\alpha$ and ERK1/2, and down-regulates $C O L 2 A 1$ in a G-protein-dependent manner. a Detection of phosphorylated and total CaMKII $\alpha$ in primary chondrocytes treated with Wnt$3 a$ or vehicle for different time points. $\beta$-Actin was used as a loading control $(n=3)$. b

Calcium mobilization in primary chondrocytes pre-treated by the G-protein inhibitor PTX or vehicle and then with Wnt-3a $(n=3)$. (c) Detection of phosphorylated CaMKII $\alpha$ (pCaMKII $\alpha$ ) and phosphorylated ERK1/2 (p-ERK1/2) and total CaMKII $\alpha$ and ERK1/2 in primary chondrocytes pretreated with PTX or vehicle and treated with Wnt-3a. $\beta$-Actin was used as a loading control $(n=3)$. d Fold changes of COL2A1 expression normalized to control in primary chondrocytes pre-treated with PTX or vehicle and treated with Wnt-3a $(n=3)$. CaMKII $\alpha$ induced the activation of B-raf/ ERK1/2 signaling pathway. e Detection of p-CaMKII $\alpha$, phosphorylated B-raf (p-B-raf) and $\mathrm{p}$-ERK1/2 and of total CaMKII $\alpha$, B-raf, and ERK1/2 in primary chondrocytes pretreated with KN93, KN92, or vehicle and treated with Wnt-3a. $\beta$-Actin was used as a loading control $(n=3)$. f Fold changes of $C O L 2 A 1$ expression normalized to control in primary chondrocytes pre-treated with KN93, KN92, or vehicle and treated with Wnt-3a $(n=3)$. Data are representative of at least three experiments. Q-PCR values were normalized for the housekeeping gene ribosomal protein S29 and are expressed as relative expression compared with control. Data are expressed as mean \pm S.D. Statistical analysis was performed with an unpaired Student's $t$ test $(* p<0.05 ; * * p<0.01)$
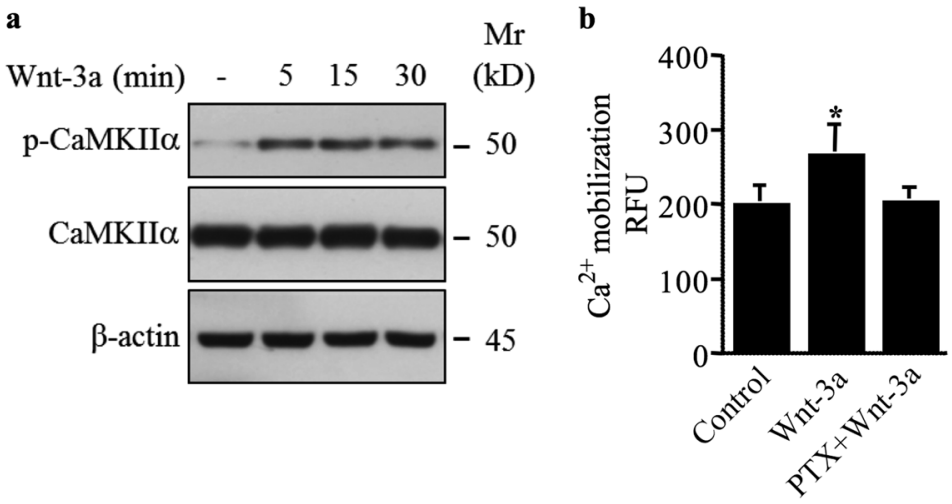

c

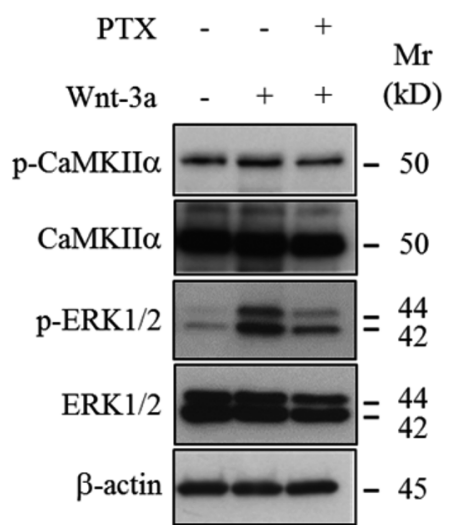

d
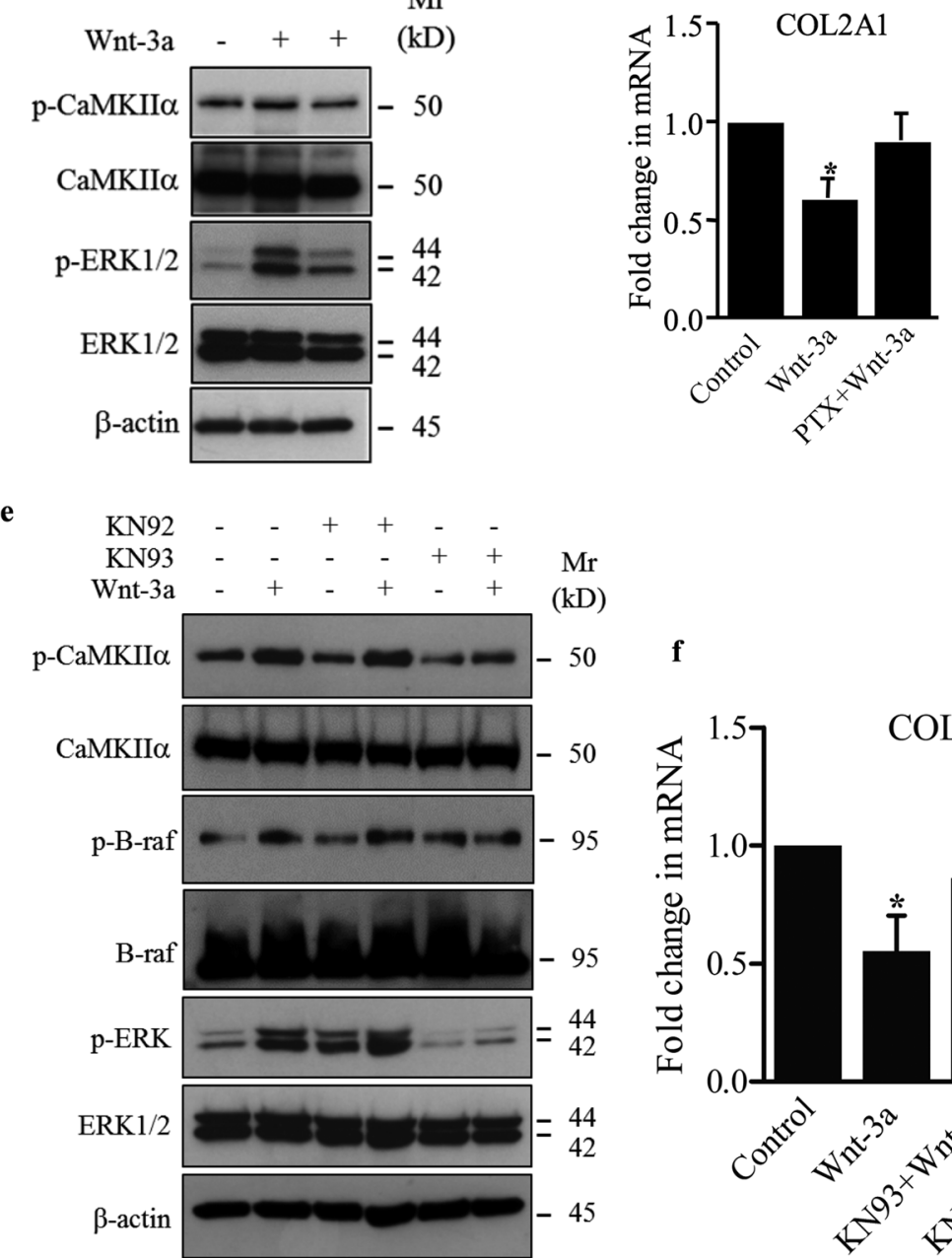

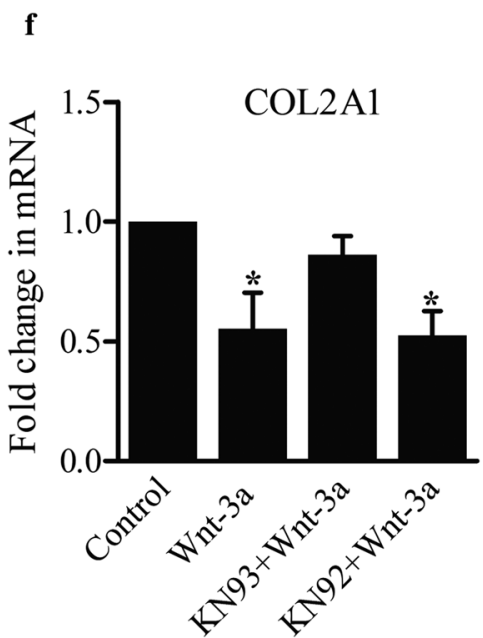

analyzed the phosphorylation status of CaMKII $\alpha$ and found that treatment with Wnt-3a induced the phosphorylation of CaMKII $\alpha$ (p-CaMKII $\alpha$ ) (Fig. 4a). To explore whether calcium mobilization is involved in the activation of ERK1/2 pathway by Wnt-3a, intracellular $\mathrm{Ca}^{2+}$ accumulation was monitored in primary chondrocytes treated or not with Wnt-3a. The results showed that Wnt-3a induced $\mathrm{Ca}^{2+}$ mobilization which was efficiently blocked by pertussis toxin (PTX), a G-protein inhibitor (Fig. 4b). Western blot analysis of the phosphorylation status of CaMKII $\alpha$ and ERK1/2 showed that PTX markedly reduced Wnt-3ainduced activation of both CaMKII $\alpha$ and ERK1/2 (Fig. 4c). In line with this, PTX prevented down-regulation of COL2Al by Wnt-3a (Fig. 4d). These results suggest that 
Wnt-3a induces loss of chondrocyte phenotype by activation of $\mathrm{Ca}^{2+} / \mathrm{CaMKII} \alpha / \mathrm{B}-\mathrm{raf} / \mathrm{ERK} 1 / 2$ signaling pathway in a G-protein-dependent manner.

Next, we examined the role of CaMKII $\alpha$ in Wnt-3ainduced activation of B-raf. To this end, chondrocytes were pre-treated or not with CaMKII inhibitor KN93 or with KN92, an analog of KN93, which does not inhibit CaMKII, and then stimulated with Wnt-3a. Analysis of cell extracts showed that not only KN93 prevented Wnt-3a-induced activation of CaMKII $\alpha$ but also prevented the activation of B-raf and almost completely abrogated the activation of ERK1/2 signaling by Wnt-3a (Fig. 4e). In contrast, KN92 did not prevent the activation by Wnt-3a of either CaMKII $\alpha$, B-raf, or ERK1/2 (Fig. 4e). These results indicate that activation of B-raf and ERK1/2 signaling is CaMKII $\alpha$ dependent, and consequently de-differentiation of chondrocytes induced by Wnt-3a is mediated by CaMKII $\alpha$. In line with this, inhibition of CaMKII 0 by KN93 prevented down-regulation of COL2Al by Wnt-3a, whereas treatment with KN92 did not (Fig. 4f).

\section{SYND4 docks CaMKIla and interacts with DVL-2 to induce Wnt/ERK1/2 signaling and chondrocyte de- differentiation}

To determine the role of SYND4 in non-canonical Wnt pathway in primary chondrocytes, we performed loss of function (Fig. 5a) and examined whether knockdown of SYND4 affects the activation of ERK1/2 signaling by Wnt3a. As shown in Fig. 5b, knockdown of SYND4 prevents the activation of B-raf and ERK1/2 by Wnt-3a, but did not significantly affect the phosphorylation of CaMKII $\alpha$ in response to Wnt-3a. These results suggest that activation of B-raf and ERK1/2 by Wnt-3a is SYND4-dependent, whereas activation of CaMKII $\alpha$ is not. Interestingly, knockdown of DVL-2 strongly reduced the activation of both B-raf/ERK1/2 and CaMKII $\alpha$ by Wnt-3a (Fig. 5b) arguing for a dependence of CaMKII $\alpha$ activation by Wnt-3a on DVL-2 but not on SYND4.

Given that activation of B-raf and ERK1/2 by Wnt-3a is both SYND4- and CaMKII $\alpha$-dependent, we hypothesized that SYND4 may relay Wnt signaling to B-raf and ERK1/2 through interaction with CaMKII $\alpha$. Coimmunoprecipitation assays were performed using extracts from cells that have been transiently transfected with HASYND4. The results clearly show that HA-SYND4 physically interacts with p-CaMKII $\alpha$ (Fig. 5c). Interestingly, the interaction between SYND4 and CaMKII $\alpha$ was significantly enhanced in the presence of Wnt-3a (Fig. 5c), suggesting that SYND4 interacts with CaMKII $\alpha$ in a Wnt3a-dependent manner. On the other hand, a potential interaction between SYND4 and DVL-2 was examined using extracts from chondrocytes that have been transfected with Flag-DVL-2 and HA-SYND4. Interestingly, DVL-2 was co-immunoprecipitated with SYND4 (Fig. 5d), suggesting that SYND4 interacts with DVL-2. SYND4 contains a transmembrane (TM) domain and a cytoplasmic (CT) domain and it is known that SYND4 normally transduces signal through its CT domain [10, 24], we sought to determine whether deletion of the CT domain of SYND4 (SYND4 $\Delta \mathrm{CT}$ ) affects the Wnt-3a-mediated activation of Braf/ERK1/2 and CaMKII $\alpha$. As shown in Fig. 5e, Wnt-3a failed to activate B-raf and ERK1/2 signaling in chondrocytes expressing SYND4 $\Delta C T$, whereas activation of CaMKII $\alpha$ is not affected. Taken together, these findings suggest that the CT domain of SYND4 is required for Wnt3a-induced activation of B-raf and ERK1/2 through interaction of its CT domain with CaMKII $\alpha$.

\section{CaMKIla phosphorylates B-raf}

We showed above that phosphorylation of B-raf by Wnt-3a depends on CaMKII $\alpha$. Might B-raf be substrate for CaMKII $\alpha$ ? To investigate this hypothesis, we examined whether CaMKII $\alpha$ is able to physically interact with B-raf by performing co-immunoprecipitation experiments. We stimulated chondrocyte cells with Wnt-3a and then performed immunoprecipitation with anti-B-raf-specific antibodies. As shown in Fig. 6a, p-CaMKII $\alpha$ interacts with B-raf and this interaction was markedly increased by Wnt-3a. To further confirm this interaction, we conducted coimmunoprecipitation experiments in human embryonic kidney 293 (HEK293) cells that express Flag-B-raf or transfected with empty vector and subjected both to immunoprecipitation with anti-Flag antibodies. After Western blot analysis for CaMKII $\alpha$ on these immunoprecipitates, we found that endogenous CaMKII $\alpha$ associates with the anti-B-raf immunoprecipitation complex from cells transfected with Flag-B-raf-expressing vector (Fig. 6b), indicating that CaMKII $\alpha$ associates with B-raf.

Noteworthy, the Ser445 residue of B-raf could be a potential phosphorylation site for CaMKII based on the consensus substrate recognition motif Hyd-X-R-NB-X-S/THyd for CaMKII [25] (Fig. 6c). An in vitro kinase assay with a glutathione $S$-transferase (GST) fusion protein containing B-raf kinase domain (amino acids 417-766) expressed in E. coli and purified by immunoaffinity (Fig. 6d-e) together with CaMKII $\alpha$ and $\left[\gamma^{32}\right.$ P]ATP revealed that B-raf was robustly phosphorylated by CaM$\mathrm{KII} \alpha$, as assessed by radiography (Fig. 6f). To determine whether the Ser445 was the phosphorylation site, we generated point mutation of Ser445 and discovered that mutating Ser445 to alanine (S445A) markedly reduced phosphorylation by CaMKII $\alpha$ (Fig. 6f). Of note, mutation of both the Ser445 and Ser446 residues to alanine (S445/ 446A) reduces further the phosphorylation of B-raf 
Fig. 5 Syndecan 4 regulates Wnt-3a-induced activation of ERK1/2 signaling and interacts with CaMKII $\alpha$ and DVL-2. a Fold changes in the mRNA expression of SYND4 in primary chondrocytes transfected with siRNA to SYND4 (Si-SYND4) or siRNA control (Si-control) evaluated by Q-PCR $(n=4)$. b Detection of phosphorylated B-raf (p-B-raf), phosphorylated CaMKII $\alpha$ (pCaMKII $\alpha$ ), and phosphorylated ERK1/2 (p-ERK1/2), and of total B-raf, CaMKII $\alpha$, and ERK1/2 in cell lysates of primary chondrocytes transfected with siRNA specific to SYND4 (Si-SYND4), DVL-2 (Si-DVL-2), or siRNA control (Si-control). $\beta$-Actin was used as a loading control $(n=3)$. $\mathbf{c}$ Detection of p-CaMKII $\alpha$ and HA-SYND4 in cell lysates of primary chondrocytes, transfected with either empty vector (Native) or HA-SYND4expressing vector and treated with Wnt-3a or vehicle, after immunoprecipitation with antiHA antibodies. Total cell lysates were analyzed by immunoblot with anti-p-CaMKII $\alpha$ and antiHA antibodies. $\beta$-Actin was used as a loading control $(n=$ 3). d Detection of HA-SYND4 and Flag-DVL-2 in cell lysates of primary chondrocytes, transfected with empty vector (Native) or HA-SYND4expressing or Flag-DVL-2expressing vectors, after immunoprecipitation with antiFlag antibodies. Total cell lysates were analyzed by immunoblot with anti-HA and anti-Flag antibodies. $\beta$-Actin was used as a loading control $(n=3)$. e Detection of p-B-raf, p-ERK1/2, and p-CaMKII $\alpha$, and of total B-raf, ERK1/2, and CaMKII $\alpha$ in cell lysates of primary chondrocytes transfected either with empty vector (Native) or HASYND4 $\triangle \mathrm{CT}$ vector expressing SYND4 deleted from the CT domain and treated with Wnt-3a or vehicle. $\beta$-Actin was used as a loading control $(n=3)$

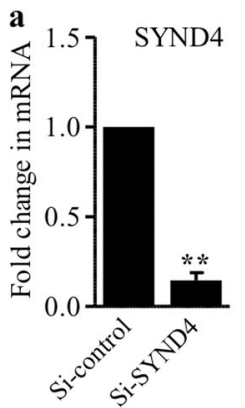

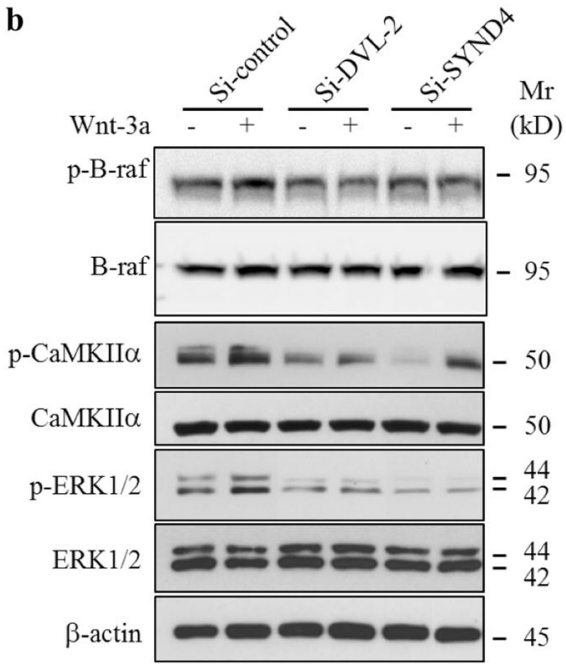

d

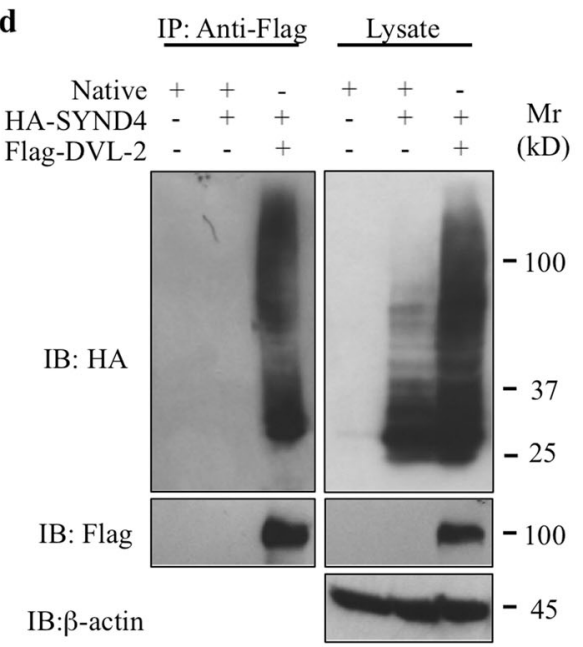

c

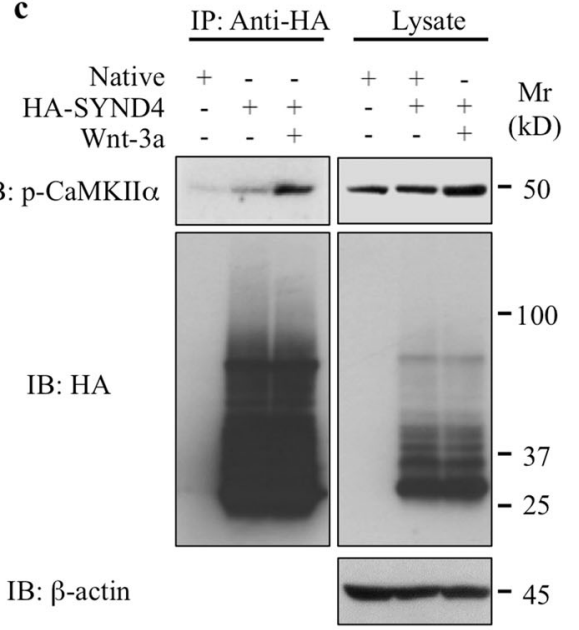

e

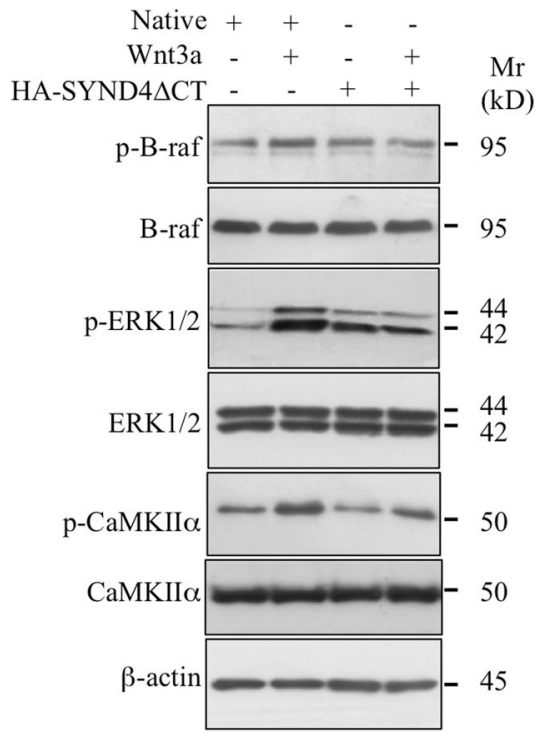


Fig. 6 CaMKII $\alpha$ associates and phosphorylates B-raf at Ser445. a Detection of p-CaMKII $\alpha$ and B-raf in cell lysates of primary chondrocytes treated with Wnt$3 \mathrm{a}$ or vehicle after immunoprecipitation with antiB-raf antibodies. Total cell lysates were analyzed by immunoblot with anti-pCaMKII $\alpha$ and anti-B-raf antibodies. $\beta$-Actin was used as a loading control $(n=3)$. b Detection of p-CaMKII $\alpha$ and Braf in the lysate of HEK293 cells transfected with empty vector (Native) or Flag-B-rafexpressing vector after immunoprecipitation with Flag antibodies. Total cell lysates were analyzed by immunoblot with anti-Flag and anti-pCaMKII $\alpha$ antibodies. $\beta$-Actin was used as a loading control $(n=3)$. c Sequence of B-raf peptides (438-453) containing the CaMKII $\alpha$ recognition sequence RXXS. The CaMKII $\alpha$ phosphorylation site is shown in red. d Brilliant Blue protein staining of purified GST fusion wild-type and B-raf mutants expressed in E. coli. Arrow indicates non-specific protein. e Detection of GST fusion wildtype and B-raf mutant proteins purified from recombinant bacterial extracts using antiB-raf antibodies. f

Autoradiography analysis of GST fusion proteins that were incubated with activated CaMKII $\alpha$ in the presence of $\left[\gamma-{ }^{32} \mathrm{P}\right]$ ATP $\left({ }^{32} \mathrm{P}\right) . \mathbf{g}$ Immunoblot analysis with phospho-B-raf (S445) antibodies of GST wildtype and B-raf mutants that were phosphorylated in vitro (p-B-raf) with activated CaMKII $\alpha$, unactivated CaMKII $\alpha$ was used as control. Total B-raf was detected using anti-B-raf antibodies. $\mathbf{h}$ Detection of phospho-B-raf and total B-raf and CaMKII $\alpha$ in primary chondrocytes transfected with empty vector (Native), B-rafexpressing vector, or co-

transfected with B-raf and CaMKII $\alpha$ (T286D) or with B-raf S445-expressing and CaMKII $\alpha$ expressing (T286D) vectors. Immunoblots were probed with anti-phospho-B-raf (S445), Braf, or CaMKII $\alpha$. $\beta$-Actin was used as a loading control $(n=3)$ a b

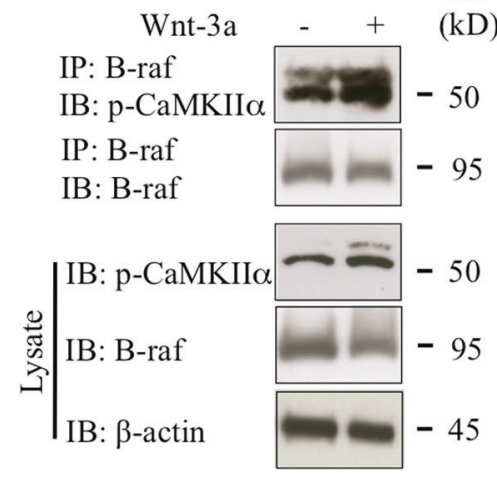
$\mathrm{Mr}$

IP: Flag
IB: p-CaMKII $\alpha$
IP: Flag
IB: Flag

Native Flag-B-raf

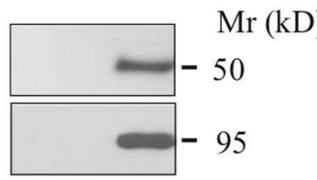

\begin{tabular}{l|l} 
IB: $p$-CaMKII $\alpha$ \\
IB: Flag \\
IB: $\beta$-actin
\end{tabular}

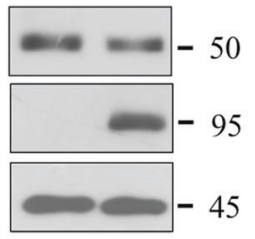

c

CaMKII site

B-raf ${ }^{438}{ }^{K T L G R R D S S D D W E I P D ~}{ }^{453}$ d

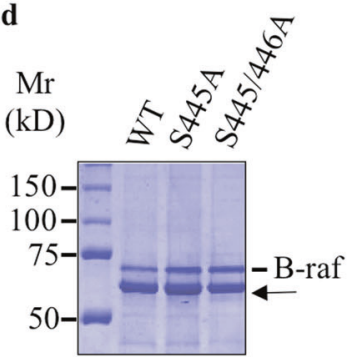

e

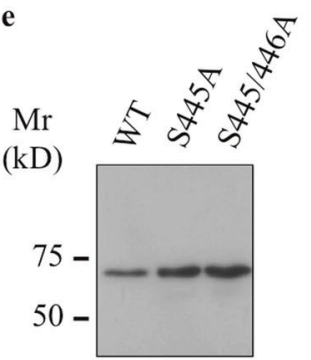

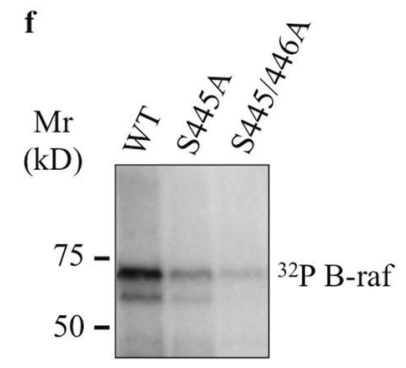

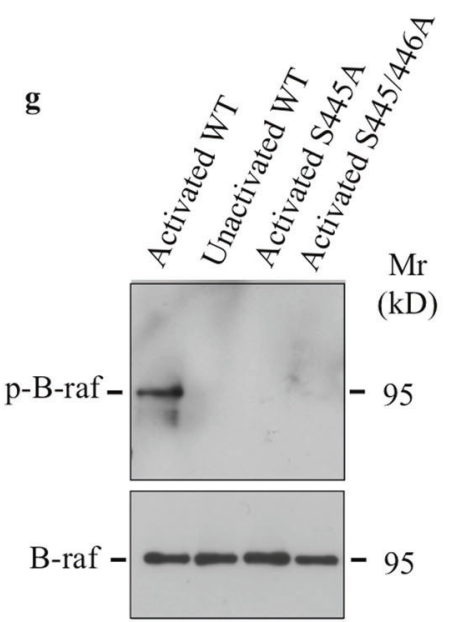

h

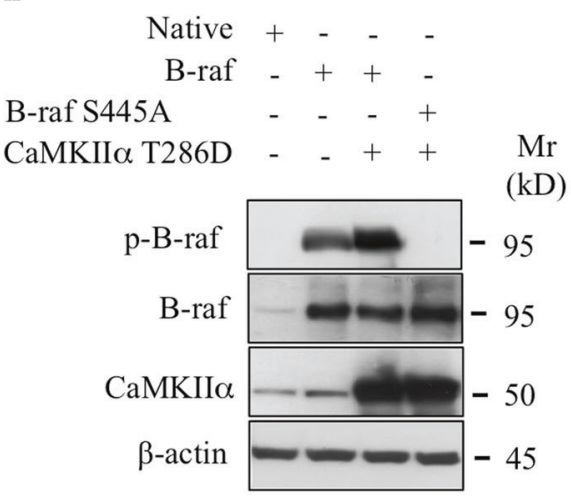


(Fig. 6f). To further confirm these results, we tested whether phospho-specific antibodies to Ser445 are able to bind to the activated B-raf. A kinase assay with CaMKII $\alpha$, ATP, and Braf or B-raf (S445A) followed by Western blot revealed that the antibodies bind only to activated B-raf but not to B-raf mutants S445A and S445/446A or unactivated B-raf (Fig. 6g). To test whether phosphorylation of B-raf is modulated by CaMKII in intact cells, we transfected wildtype B-raf or B-raf S445A in HEK293 cells, and native vector was used as the control. Immunoblots of cell lysates probed with phospho-specific antibodies to S445 indicated that B-raf was phosphorylated at Ser445 under basal conditions (Fig. 6h). However, after co-transfection with constitutively active form of CaMKII $\alpha$ (T286D), the basal-level phosphorylation of B-raf was significantly increased (Fig. 6h), whereas no signal was observable with phosphodeficient mutant (Fig. 6h). Taken together, these results suggest that B-raf is phosphorylated at Ser445 residue by CaMKII $\alpha$.

\section{Frizzled 6-mediated non-canonical Wnt-3a/ERK1/ 2 signaling and de-differentiation of chondrocytes}

Frizzled $(\mathrm{Fz})$ is a family of G-protein-coupled receptor (GPCR) proteins that serves as receptors in the Wnt signaling pathway. To address the question of which of the known FZD genes could be involved in Wnt-3a activation of Wnt non-canonical pathway, we first performed quantitative real-time PCR (qRT-PCR) to monitor the expression pattern of all genes encoding Wnt receptors in human primary chondrocytes. The result obtained showed that Fz-6 and Fz-8 are predominantly expressed in chondrocytes compared to other Fz receptors (Fig. 7a). Given that noncanonical Wnt-3a is characterized by the activation of CaMKII $\alpha$ and ERK1/2 and the inhibition of COL2A 1 in the human chondrocytes, we evaluated the impact of Fz-6 deficiency on this signaling pathway. The knockdown of Fz-6 efficiently decreased its expression (Fig. 7b) and did not impair Wnt-3a-induced up-regulation of the canonical Wnt/ $\beta$-catenin downstream target gene AXIN2 (Fig. 7c). However, knockdown of Fz-6 abrogated Wnt-3a-induced down-regulation of the non-canonical Wnt downstream target gene COL2A1 (Fig. 7d). These data suggest that Fz-6 is not required for the activation of Wnt-3a canonical signaling, but essential for the activation of non-canonical signaling in chondrocytes. To further confirm these results, we tested whether the knockdown of Fz-6 affects Wnt-3ainduced stabilization of $\beta$-catenin and found that it has no significant effect (Fig. 7e). Next, we examined whether silencing of Fz-6 effects the activation of non-canonical ERK1/2 signaling. Indeed, knockdown of Fz-6 prevents the activation of ERK1/2 signaling by Wnt-3a and abrogates the phosphorylation of CaMKII $\alpha$ (Fig. 7f). These results indicate that activation of CaMKII $\alpha$ and ERK1/2 signaling is no more modulated by Wnt-3a in chondrocytes that are Fz-6-deficient. Taken together, these observations support the conclusion that Fz-6 mediates non-canonical Wnt-3ainduced de-differentiation of chondrocytes through activation of CaMKII $\alpha / \mathrm{B}-\mathrm{raf} / \mathrm{ERK} 1 / 2$ cascade.

\section{Discussion}

Loss of phenotypic stability of articular chondrocytes has been proposed as an early event in the onset of OA disease [26]. De-differentiation of chondrocytes occurs through a programme with similarities to terminal differentiation of chondrocytes (hypertrophy), as can be found in the growth plate [27]. This triggers a disturbed cartilage homeostasis favoring degenerative changes [27, 28]. Wnt signaling has a pivotal role in regulating chondrocyte differentiation during endochondral ossification and was also shown to play a regulative role in OA cartilage [7, 29], leading to chondrocyte hypertrophic differentiation. Here, we demonstrated that Wnt-3a-induced loss of chondrocyte phenotype is mediated by Fz-6 through $\mathrm{Ca}^{2+} / \mathrm{CaMKII} \alpha$-dependent activation of B-raf/ERK1/2 signaling pathway (Fig. 7g). Indeed, knockdown of ERK1/2 or chemical inhibition of its activation prevented Wnt-3a-induced hypertrophic dedifferentiation of chondrocytes, which is associated with down-regulation of $C O L 2 A 1$ [30, 31], indicating that activation of ERK1/2 is a key event in Wnt-3a-induced loss of chondrocyte phenotype.

An important issue we addressed here is the mechanism of activation of ERK1/2 pathway by non-canonical Wnt signaling, which promotes chondrocyte de-differentiation. It has been shown that SYND4 is highly expressed in hypertrophic chondrocytes during embryonic development and during OA, suggesting a role in chondrocyte dedifferentiation. Interestingly, loss of SYND4 in mice protects from development of OA-like disease [13]. Accordingly, we showed that knockdown of SYND4 reduced basal activation of B-raf/ERK1/2 signaling and impaired its activation by Wnt-3a in human primary chondrocytes. SYND4 has been shown to interact with different partners through its CT domain [11]. Interestingly, coimmunoprecipitation studies revealed, for the first time, that SYND4 interacts with p-CaMKII $\alpha$ in a Wnt-dependent manner. On the other hand, co-immunoprecipitation studies revealed that DVL-2 docks B-raf in Wnt-dependent manner; therefore, in the presence of Wnt ligand, interaction between DVL-2 and SYND4 may promote assembly of CaMKII $\alpha$ and B-raf complex necessary to relay Wnt-3a activation of ERK1/2 signaling pathway. In this case, exploring whether B-raf can directly be activated by CaMKII $\alpha$ and transduces signals to MEK and ERK1/2 
a
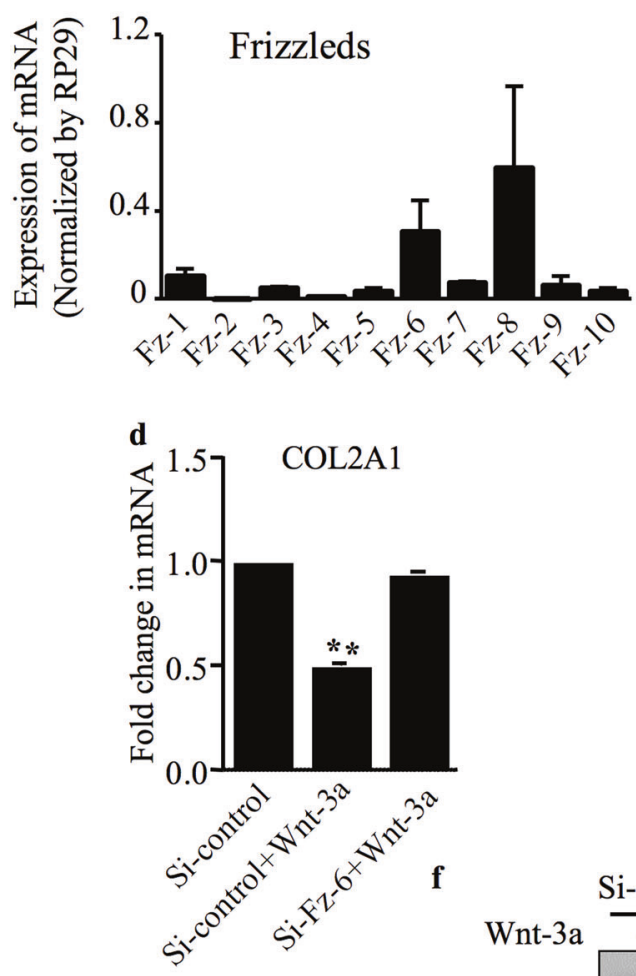

\section{b}

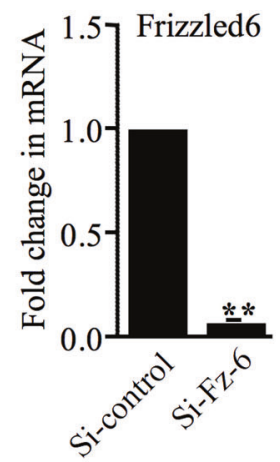

c

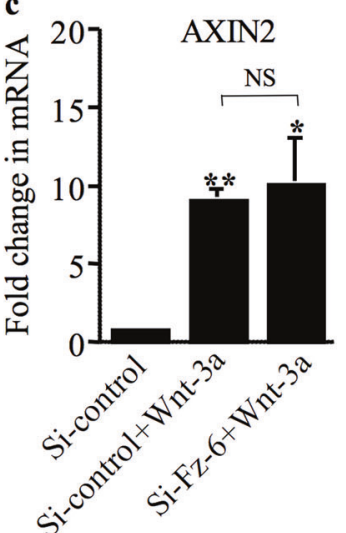

e

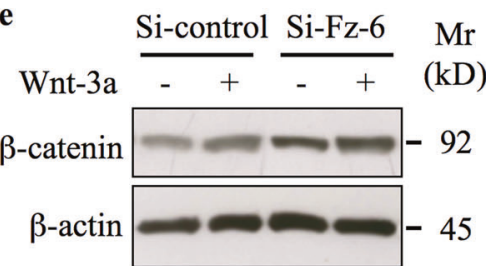

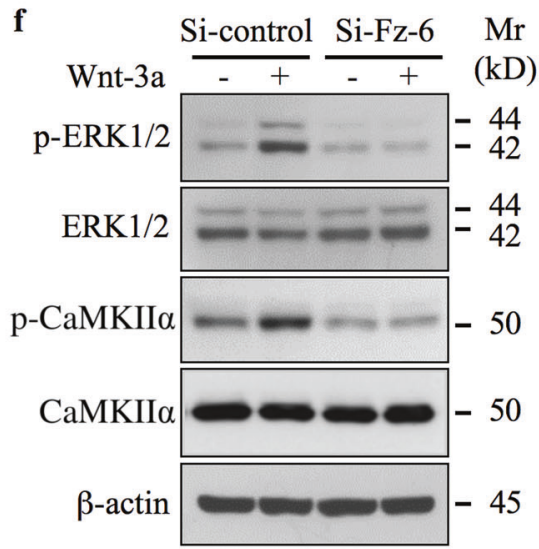

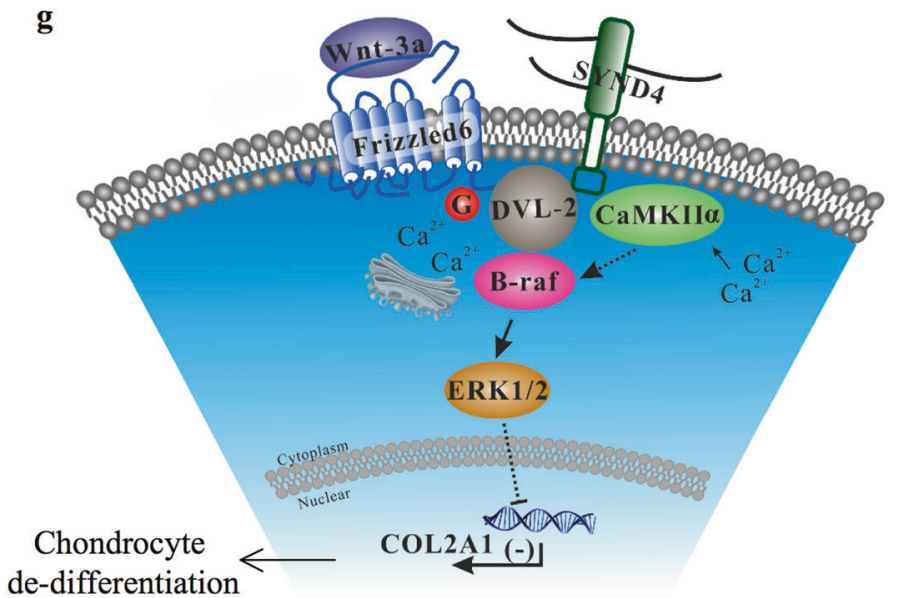


Fig. 7 Frizzled 6 mediates non-canonical Wnt-3a-induced loss of chondrocyte phenotype. a Expression of different $\mathrm{Fz}$ receptors was evaluated by absolute Q-PCR. Specific DNA segment for each of the $10 \mathrm{Fz}$ receptors (standards) was amplified by RT-PCR, purified on agarose gel, quantified, and used as template for Q-PCR. Absolute quantification was determined compared to standards that are run in parallel. Data are expressed as mean \pm S.D. of three separate experiments. b Fold changes in the expression of Fz-6 in primary chondrocytes transfected with siRNA specific to Fz-6 (Si-Fz-6) or siRNA control (Si-control) $(n=5)$. c Fold changes in the expression of the Wnt canonical target gene AXIN2 (d) and of the chondrocyte phenotypic marker $C O L 2 A 1$ in primary chondrocytes that were transfected with siRNA specific to Fz-6 (Si-Fz-6) or siRNA control ( $\mathrm{Si}$-control) and treated or not with Wnt-3a $(n=3)$. e Western blotting for $\beta$ catenin in chondrocytes transfected with Si-Fz-6 or Si-control and treated with Wnt-3a or vehicle. $\beta$-Actin was used as a loading control $(n=3)$. f Detection of phosphorylated ERK1/2 (p-ERK1/2) and phosphorylated CaMKII $\alpha$ (p-CaMKII $\alpha$ ), and total ERK1/2 and CaMKII $\alpha$ in primary chondrocytes transfected with siRNA control (Sicontrol) or siRNA to Fz-6 (Si-Fz-6) and treated with Wnt-3a or vehicle. $\beta$-Actin was used as a loading control $(n=3)$. g A working model for signaling regulation by non-canonical Wnt in chondrocytes. See text for details. Q-PCR values were normalized for the housekeeping gene ribosomal protein S29 and are expressed as relative expression compared with control. One representative blot of three independent experiments is shown. Data are expressed as mean \pm S.D. Statistical analysis was performed with an unpaired Student's $t$ test (NS, statistically not significant; $* p<0.05 ; * * p<0.01$ )

MAPK is an important issue. Our findings show that CaMKII $\alpha$ phosphorylates B-raf in vitro, in heterologous cells and in primary chondrocytes. Moreover, overexpression of constitutively active CaMKII $\alpha$ in heterologous cells results in a substantial increase in B-raf phosphorylation. Therefore, revealing for the first time that CaMKII $\alpha$ phosphorylates B-raf.

Wnt-3a is known to activate both canonical and noncanonical Wnt signaling. One of the key questions is whether individual $\mathrm{Fz}$ receptors can activate both pathways, or if the simultaneous activation of canonical and CaMKII $\alpha$ / ERK1/2 pathways is mediated through $\mathrm{Fz}$ receptors with different signaling specificity. We found that Fz-6 and Fz-8 are the most expressed Fz receptors in human chondrocytes. It has been shown that Fz-6 did not activate the canonical Wnt pathway following exposure to various Wnts, including Wnt-3a in HEK293 cells [32]. We have examined the role of the Wnt signaling receptor Fz-6 and demonstrated that Fz-6 does not affect the activation of $\beta$-catenin pathway as monitored by stabilization of $\beta$-catenin and expression of the target gene AXIN2. In contrast, Fz-6 is critical for the activation of non-canonical pathway as demonstrated by the phosphorylation status of CaMKII $\alpha$ and ERK1/2, and down-regulation of COL2A1 following knockdown of Fz-6.

$\mathrm{Fz}$ proteins possess seven TM domains and their signaling depend on heterotrimeric $G$ proteins. Fzs are pre-

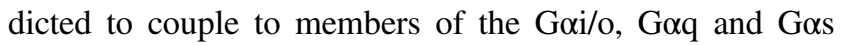

families of $\mathrm{G}$ proteins, with Fz-6 predicted to interact solely with members of Goi/o family [33]. In mammalian cells, Wnt-3a elicited Fz-dependent guanine-nucleotide exchange on Go/i proteins, which is sensitive to PTX [34]. We showed that inactivation of members of the Goi/o family with PTX, which decouples the Go/i proteins from their receptors, markedly reduced Wnt-3a-induced activation of CaMKII $\alpha$ and ERK1/2 signaling and prevented downregulation of $C O L 2 A 1$ by Wnt-3a, indicating that Fz-6 acts as Go/i-coupled GPCRs as suggested by Koval and Katanaev [34]. More recently, it has been shown that Fz-6

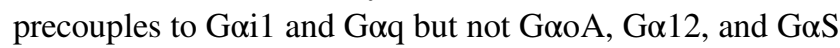
[35]. Goi/o-coupled GPCRs typically reduce cAMP levels through Goi/o subunits and induce changes in intracellular calcium [36]. We found that increasing the level of cAMP by forskolin did not prevent the inhibition of COL2A1 expression by Wnt-3a (data not shown), suggesting that cAMP pathway is not the main mechanism involved in Wnt-3a-induced down-regulation of COL2A1, although we cannot exclude a role by cAMP in this process.

Healthy cartilage is extremely difficult to obtain for ethical reason. In this study, we have used cartilage samples that are histological and macroscopically normal; however, this has a limitation as we cannot exclude that they were subjected to paracrine effects from neighboring disease compartment.

In summary, our study introduces a compelling new mechanism of Wnt-induced activation of ERK1/2 signaling and chondrocyte de-differentiation, which is essential in both normal development and OA pathogenesis. As dedifferentiation of chondrocyte is an important issue in OA disease, our study provides new potential targets such Fz-6, B-raf, and CaMKII $\alpha$ for the development of anti-OA drugs.

\section{Materials and methods}

\section{Chondrocytes and cartilage explants isolation and culture}

Human primary chondrocytes were isolated from cartilage obtained from patients who underwent joint replacement for knee OA. Cartilage tissue was dissected from preserved areas of the femoral condyles and patellar groove avoiding subchondral bone and fibrocartilage. Cartilage was washed twice with Dulbecco's Modified Eagle's Medium/Nutrient Mixture F12 (DMEM F12) complete medium (2 mM glutamine, $100 \mu \mathrm{g} / \mathrm{ml}$ streptomycin, $100 \mathrm{IU} / \mathrm{ml}$ penicillin, and $10 \%(\mathrm{v} / \mathrm{v})$ fetal bovine serum) and the aliquot was then digested with pronase $(1 \mathrm{mg} / \mathrm{ml})$ for $30 \mathrm{~min}$ at $37^{\circ} \mathrm{C}$ followed by an overnight digestion in collagenase $\mathrm{B}(1 \mathrm{mg} / \mathrm{ml})$ in a similar medium as above. The chondrocytes recovered from the digestion were seeded at a density of 25,000 cells/ $\mathrm{cm}^{2}$ in DMEM F12 complete medium at $37^{\circ} \mathrm{C}$ in a 
humidified atmosphere supplemented with $5 \% \mathrm{CO}_{2}$. All experiments were performed using confluent $\mathrm{P} 0$ cells.

For cartilage explant isolation, cartilage specimens were dissected within $1 \mathrm{~h}$ of operation, and full-thickness cartilage was removed from preserved areas of the femoral condyles using a $6 \mathrm{~mm}$ biopsy punch. Extreme care was taken to avoid subchondral bone, osteophytic cartilage, and fibrocartilage. Explants were washed with DMEM F12 complete medium and cultured in 24-well plates in the same medium and condition as chondrocytes for $48 \mathrm{~h}$ before use.

Aliquot of cartilage was used for histological scoring for features of OA as described previously [37]. Only cells and explants obtained from samples with a Mankin score $<4$ were used for subsequent experiments. This study was approved by our local research institution (Commission de la Recherche Clinique; registration no. UF 9757, CPRC 2004, Cellules souches et chondrogénèse). The protocol conforms to the ethical guidelines of the Declaration of Helsinki, and written informed consent has been obtained from each patient.

\section{Cell and cartilage explant treatments}

Human primary chondrocytes were seeded onto six-well plates at $5 \times 10^{5}$ cells/well and allowed to attach overnight in standard culture conditions. The cells were then treated for $12 \mathrm{~h}$ with $100 \mathrm{ng} / \mathrm{ml}$ of recombinant Wnt-3a (R\&D Systems, Minneapolis, MN, USA) or vehicle $(0.1 \%$ bovine serum albumin (BSA) in phosphate-buffered saline (PBS)) in DMEM F12 medium ( $2 \mathrm{mM}$ glutamine, $100 \mu \mathrm{g} / \mathrm{ml}$ streptomycin, $100 \mathrm{IU} / \mathrm{ml}$ penicillin, and $2 \%(\mathrm{v} / \mathrm{v})$ fetal bovine serum) prior to gene or protein expression analyses. When co-treatments with inhibitors and Wnt-3a are indicated, cells were pre-treated for $30 \mathrm{~min}$ with the inhibitor, that is, U0126 (10 $\mu \mathrm{M}$; Cell Signaling, Danvers, CO, USA), DKK1 (100 ng/ml; R\&D Systems), and KN93 and KN92 $(10 \mu \mathrm{M}$; R\&D Systems) prior to treatment with Wnt-3a or vehicle.

\section{Gene expression analysis}

Total RNA from chondrocytes and cartilage explants was extracted using TRIzol (Lifetech, Carlsbad, CA, USA) and purified with RNeasy kit (Qiagen, Hilden, Germany) according to the manufacturer's instructions. The reverse transcription was performed using $500 \mathrm{ng}$ (primary chondrocytes) or $200 \mathrm{ng}$ (cartilage explants) of total RNA from each sample with iScript Ready to use cDNA supermix (Bio-Rad, Hercules, CA, USA). Quantitative PCR was performed with iTaq ${ }^{\mathrm{TM}}$ Universal SYBER Green Supermix kit (Bio-Rad, Hercules, CA, USA) using StepOnePlus ${ }^{\mathrm{TM}}$ Real-Time PCR Systems (Applied Biosystems). Primer sequences are listed in Table $\mathrm{S} 1$.

\section{Plasmids and siRNA}

B-raf vector was a gift from Dustin Maly (University of Washington, Seattle, WA, USA), Flag-DVL-2 and Flag-DVL-2( $\Delta 361-736)$ vectors were a gift from Jeff Wrana (University of Toronto, Toronto, ON, Canada), and TOP-FLASH-firefly reporter plasmid was a gift from Randall Moon (University of Washington). B-raf V600E and Braf D594V mutants were generated by site-directed mutagenesis with B-raf as template using QuikChange SiteDirected Mutagenesis kit (Agilent, Santa Clara, CA, USA). HA-SYND4, HA-SYND4 $\Delta C T$, and Flag-B-raf were generated by PCR and cloned into EcoRI and BamHI or SmaI and PstI sites of pCMV empty vector (Stratagene, Valencia, CA, USA). GST C-tail constructs were amplified with synthetic primers containing $N c o \mathrm{I}$ and $N o t \mathrm{I}$ flanking regions from B-raf and mutant plasmids and subcloned into the GST fusion vector pET41 (Novagene) to generate pET-B$\operatorname{raf} \Delta \mathrm{NT} / \mathrm{GST}, \mathrm{pET}-\mathrm{B}-\mathrm{raf} \Delta \mathrm{NT}-\mathrm{S} 465 \mathrm{~A}$, and pET-B-raf $\Delta \mathrm{NT}$ S465/466A bacterial vectors expressing the wild-type and mutants of the B-raf kinase domain (amino acids 417-766) in fusion with GST. All the vectors were verified by sequencing. Small interfering RNA (siRNA) targeting SYND4, MAPK1, MAPK3, DVL-1, DVL-2, DVL-3, CTNNB1, Fz-6, and control siRNA were purchased from Qiagen and are listed in Table S2.

\section{Transfection}

Subconfluent human primary chondrocytes in 6-well plates were transfected in triplicate with $2 \mu \mathrm{g}$ of plasmid using Lipofectamine 2000 transfection reagent (Invitrogen) according to the manufacturer's instructions. For siRNA transfection, cells were transfected with $25 \mathrm{nM}$ siRNAs using DharmaFECT transfection reagent (Thermo Scientific, Waltham, MA, USA) according to the manufacturer's instructions. Twenty-four hours after transfection, the medium was replaced, and at $48 \mathrm{~h}$ after transfection, cells were stimulated for $12 \mathrm{~h}$ with recombinant Wnt-3a (100 ng/ $\mathrm{ml}$; R\&D Systems) or vehicle at $37^{\circ} \mathrm{C}$ with $5 \% \mathrm{CO}_{2}$.

\section{Western blotting}

Total protein from chondrocytes was extracted using RIPA buffer $(150 \mathrm{mM} \mathrm{NaCl}, 50 \mathrm{mM}$ Tris-HCl, pH 7.5, $1 \%$ deoxycholate, $0.1 \%$ SDS, $1 \%$ Triton X-100) supplemented with protease and phosphatase inhibitors (Roche Diagnostics, Indianapolis, IN, USA). Cell lysates were sonicated on ice and protein concentration of the samples was determined by the Bradford method. Proteins $(50 \mu \mathrm{g} / \mathrm{lane})$ were separated on $10 \%$ sodium dodecyl sulfate-polyacrylamide gel electrophoresis (SDS-PAGE) gels, transferred to a polyvinylidene fluoride membrane (Millipore, Eschborn, 
Germany), and subsequently blocked in PBS-Tween-20 containing 5\% non-fat milk or 5\% BSA. Membranes were then incubated overnight with primary antibodies directed against p44/42 MAPK, phospho-p44/42, $\beta$-catenin, DVL-2, DVL-3, CaMKII $\alpha$, phospho-B-raf (S445) (diluted 1:1000; Cell Signaling), DVL-1, phospho-CaMKII $\alpha$, B-raf (diluted 1:200; Texas, Santa Cruz, TX, USA), Flag (diluted 1:1000; Sigma, Saint Louis, MO, USA), or HA (diluted 1:1000; BioLegend, San Diego, CA, USA), followed by incubation with horseradish peroxidase-conjugated secondary antibodies (diluted 1:2000; Cell Signaling). Antibodies were diluted in $5 \%$ BSA $/ 0.01 \%$ Tween-20 in PBS. The blots were then developed using Clarity Western ECL substrate (Bio-Rad) according to the manufacturer's instruction.

\section{Immunoprecipitation}

For immunoprecipitation, subconfluent primary chondrocytes in 10-cm plates were treated with Wnt-3a (100 ng/ $\mathrm{ml}$ ) or vehicle for $1 \mathrm{~h}$ and were lysed on ice with TNE buffer (50 mM Tris, pH 8, 1\% IGEPAL ${ }^{\circledR}$ CA-630, $150 \mathrm{mM}$ $\mathrm{NaCl}, 1 \mathrm{mM}$ ethylenediaminetetraacetic acid (EDTA)) supplemented with protease and phosphatase inhibitors (Roche Diagnostics). The lysates were sonicated and centrifuged and the resultant supernatants were used to recover B-raf, and Flag-tagged and HA-tagged proteins by incubating overnight at $4{ }^{\circ} \mathrm{C}$ with anti-B-raf, anti-Flag, and antiHA antibodies, respectively, followed by incubation with protein A beads (Millipore, Eschborn, Germany) for $3 \mathrm{~h}$ at room temperature. After washing with lysis buffer, bound proteins were eluted with the loading buffer and analyzed by immunoblotting. Immunoblotting was performed as described previously with antibodies against Flag, HA, Braf, DVL-2, and p-CaMKII $\alpha$.

\section{Intracellular calcium ion changes}

Calcium mobilization induced by Wnt-3a was performed with human primary chondrocytes using the Fluo-4 NW Calcium Assay kit (Molecular Probes/Invitrogen, Carlsbad, CA, USA). Primary chondrocytes were grown on 24-well plates in the presence or absence of PTX (Sigma-Aldrich) overnight. The calcium-sensitive dye, Fluo-4 AM $(2 \mu \mathrm{M})$, was added to the cultured chondrocytes and allowed to incorporate for $1 \mathrm{~h} \quad(30 \mathrm{~min}$ at room temperature and $30 \mathrm{~min}$ at $37^{\circ} \mathrm{C}$ ). After $5 \mathrm{~min}$ of treatment with either vehicle (control) or with Wnt-3a, the fluorescence was measured with a Wallac/Perkin-Elmer Victor V2 fluorescent plate reader. Data were subtracted from background and presented as the mean of three separate experiments.

\section{GST fusion protein production and in vitro kinase assay}

GST fusion proteins were purified from BL21 bacterial cells transformed with pET-B-raf $\triangle \mathrm{NT} / \mathrm{GST}$, pET-B-raf $\triangle \mathrm{NT}$ S465A, or pET-B-raf $\triangle$ NT-S465/466A using GE Healthcare protocol. Fifty milliliters of cultures was inoculated with $0.5 \mathrm{ml}$ of overnight cultures and grown at $37^{\circ} \mathrm{C}$ until an absorbance at $600 \mathrm{~nm}\left(A_{600}\right)$ of $0.6-0.8$. Then, an osmotic stress and heat shock were induced by the addition of $0.5 \mathrm{M}$ $\mathrm{NaCl}$ and $2 \mathrm{mM}$ betaine, and incubated at $47^{\circ} \mathrm{C}$ for $1 \mathrm{~h}$. To induce protein expression, $1 \mathrm{mM}$ isopropyl $\beta$-D-1-thiogalactopyranoside was added to the cultures, and they were grown at $20{ }^{\circ} \mathrm{C}$ overnight. The cells were then lysed with a sonicator in a Tris-buffered saline containing protease inhibitors (Roche), $1 \mathrm{mM}$ EDTA, and 5\% (v/v) glycerol. The sonicated lysate was incubated with a 5:1 ratio of glutathione-sepharose 4B (GE Healthcare) overnight at $4{ }^{\circ} \mathrm{C}$ and washed extensively with PBS. For CaMKII in vitro phosphorylation, B-raf fusion proteins were phosphorylated in $50 \mathrm{mM}$ Tris- $\mathrm{HCl}(\mathrm{pH} 7.5), 10 \mathrm{mM} \mathrm{MgCl}_{2}, 0.2 \mathrm{mM}$ dithiothreitol, $0.1 \mathrm{mM}$ EDTA, $1.2 \mu \mathrm{M}$ calmodulin, $2 \mathrm{mM}$ $\mathrm{CaCl}_{2}, 200 \mu \mathrm{M}$ ATP, and $1 \mathrm{pmol}$ of $\left[\gamma_{-}{ }^{32} \mathrm{P}\right] \mathrm{ATP}(3000 \mathrm{Ci} /$ mmol) with $200 \mathrm{U}$ of recombinant activated CaMKII $\alpha$ (New England Biolabs). The in vitro kinase assays were performed at $30^{\circ} \mathrm{C}$ for $30 \mathrm{~min}$. The reactions were terminated with the addition of SDS-PAGE sample buffer and incubation at $65{ }^{\circ} \mathrm{C}$ for $5 \mathrm{~min}$. The proteins were resolved by SDS-PAGE and visualized by autoradiography. For in vitro kinase assays analyzed by immunoreactivity, SDSPAGE separated proteins were transferred to polyvinylidene difluoride membranes and assayed for phosphorylation (or total protein) by immunoblotting using anti-phospho-B-raf or anti-B-raf antibodies.

\section{Data analysis and statistical procedures}

Each experiment was repeated at least three times independently. Quantitative data were expressed as mean \pm S.D. Statistical analysis was performed with an unpaired twotailed Student's $t$ test, and effects were considered statistically significant at $* p<0.05$. One representative immunoblot of three independent experiments was shown in results.

Acknowledgements This work was supported by Agence Nationale de la Recherche (ANR: Solv-CDG) and by Région Lorraine.

\section{Compliance with ethical standards}

Conflict of interest The authors declare that they have no conflict of interest. 


\section{References}

1. MacDonald BT, Semenov MV, He X. SnapShot: Wnt/beta-catenin signaling. Cell. 2007;131:1204-1204.

2. Semenov MV, Habas R, MacDonald BT, He X. SnapShot: noncanonical Wnt signaling pathways. Cell. 2007;131:1378.

3. MacDonald BT, Tamai K, He X. Wnt/ $/$-catenin signaling: components, mechanisms, and diseases. Dev Cell. 2009;17:9-26.

4. Clevers $\mathrm{H}$. Wnt/ $\beta$-catenin signaling in development and disease. Cell. 2006;127:469-80.

5. Gao B. Wnt regulation of planar cell polarity (PCP). Curr Top Dev Biol. 2012;101:263-95.

6. Kühl M, Sheldahl LC, Malbon CC, Moon RT. $\mathrm{Ca}^{2+} /$ calmodulindependent protein kinase II is stimulated by Wnt and Frizzled homologs and promotes ventral cell fates in Xenopus. J Biol Chem. 2000;275:12701-11.

7. Nalesso G, Sherwood J, Bertrand J, Pap T, Ramachandran M, De Bari C, et al. WNT-3A modulates articular chondrocyte phenotype by activating both canonical and non-canonical pathways. J Cell Biol. 2011;193:551-64.

8. Vermeulen L, Felipe De Sousa EM, Van Der Heijden M, Cameron K, De Jong JH, Borovski T, et al. Wnt activity defines colon cancer stem cells and is regulated by the microenvironment. Nat Cell Biol. 2010;12:468-76.

9. Monroe DG, McGee-Lawrence ME, Oursler MJ, Westendorf JJ. Update on Wnt signaling in bone cell biology and bone disease. Gene. 2012;492:1-18.

10. Leonova E, Galzitskaya O. Structure and functions of syndecans in vertebrates. Biochemistry (Mosc). 2013;78:1071-85.

11. Elfenbein A, Simons M. Syndecan-4 signaling at a glance. J Cell Sci. 2013;126:3799-804.

12. Barre PE, Redini F, Boumediene K, Vielpeau C, Pujol JP. Semiquantitative reverse transcription-polymerase chain reaction analysis of syndecan-1 and-4 messages in cartilage and cultured chondrocytes from osteoarthritic joints. Osteoarthr Cartil. 2000;8:34-43.

13. Echtermeyer F, Bertrand J, Dreier R, Meinecke I, Neugebauer K, Fuerst M, et al. Syndecan-4 regulates ADAMTS-5 activation and cartilage breakdown in osteoarthritis. Nat Med. 2009;15:1072-6.

14. Nakamura Y, Nawata M, Wakitani S. Expression profiles and functional analyses of Wnt-related genes in human joint disorders. Am J Pathol. 2005;167:97-105.

15. Yun MS, Kim S-E, Jeon SH, Lee JS, Choi K-Y. Both ERK and $\mathrm{Wnt} / \beta$-catenin pathways are involved in Wnt3a-induced proliferation. J Cell Sci. 2005;118:313-22.

16. Halleskog C, Schulte G. Pertussis toxin-sensitive heterotrimeric G $\alpha \mathrm{i} / \mathrm{o}$ proteins mediate $\mathrm{Wnt} / \beta$-catenin and $\mathrm{WNT} / \mathrm{ERK} 1 / 2$ signaling in mouse primary microglia stimulated with purified WNT-3A. Cell Signal. 2013;25:822-8.

17. Logan CY, Nusse R. The Wnt signaling pathway in development and disease. Annu Rev Cell Dev Biol. 2004;20:781-810.

18 Hamblet NS, Lijam N, Ruiz-Lozano P, Wang J, Yang Y, Luo Z, et al. Dishevelled 2 is essential for cardiac outflow tract development, somite segmentation and neural tube closure. Development. 2002;129:5827-38.

19 Lee YN, Gao Y, Wang HY. Differential mediation of the Wnt canonical pathway by mammalian dishevelleds-1, -2 , and -3 . Cell Signal. 2008;20:443-52.
20 Etheridge SL, Ray S, Li S, Hamblet NS, Lijam N, Tsang M, et al. Murine dishevelled 3 functions in redundant pathways with dishevelled 1 and 2 in normal cardiac outflow tract, cochlea, and neural tube development. PLoS Genet. 2008;4:e1000259

21. Boutros M, Paricio N, Strutt DI, Mlodzik M. Dishevelled activates JNK and discriminates between JNK pathways in planar polarity and wingless signaling. Cell. 1998;94:109-18.

22. Wallingford JB, Habas R. The developmental biology of Dishevelled: an enigmatic protein governing cell fate and cell polarity. Development. 2005;132:4421-36.

23. Simons M, Gault WJ, Gotthardt D, Rohatgi R, Klein TJ, Shao Y, et al. Electrochemical cues regulate assembly of the Frizzled/ Dishevelled complex at the plasma membrane during planar epithelial polarization. Nat Cell Biol. 2009;11:286-94.

24. Horowitz A, Simons M. Phosphorylation of the cytoplasmic tail of syndecan-4 regulates activation of protein kinase $\mathrm{C} \alpha$. J Biol Chem. 1998;273:25548-51.

25. White RR, Kwon YG, Taing M, Lawrence DS, Edelman AM. Definition of optimal substrate recognition motifs of $\mathrm{Ca}^{2+}$-calmodulin-dependent protein kinases IV and II reveals shared and distinctive features. J Biol Chem. 1998;273:3166-72.

26. Haag J, Gebhard PM, Aigner T. SOX gene expression in human osteoarthritic cartilage. Pathobiology. 2008;75:195-9.

27. Dreier R. Hypertrophic differentiation of chondrocytes in osteoarthritis: the developmental aspect of degenerative joint disorders. Arthritis Res Ther. 2010;12:216.

28. Pap T, Korb-Pap A. Cartilage damage in osteoarthritis and rheumatoid arthritis-two unequal siblings. Nat Rev Rheumatol. 2015;11:606-15.

29. Ryu JH, Chun JS. Opposing roles of WNT-5A and WNT-11 in interleukin- $1 \beta$ regulation of type II collagen expression in articular chondrocytes. J Biol Chem. 2006;281:22039-47.

30. Lefebvre V, Li P, De Crombrugghe B. A new long form of Sox 5 (L-Sox5), Sox6 and Sox9 are coexpressed in chondrogenesis and cooperatively activate the type II collagen gene. EMBO J. 1998;17:5718-33.

31. Taschner MJ, Rafigh M, Lampert F, Schnaiter S, Hartmann C. $\mathrm{Ca}^{2}$ ${ }^{+} /$Calmodulin-dependent kinase II signaling causes skeletal overgrowth and premature chondrocyte maturation. Dev Biol. 2008;317:132-46.

32. Golan T, Yaniv A, Bafico A, Liu G, Gazit A. The human Frizzled 6 (HFz6) acts as a negative regulator of the canonical $\mathrm{Wnt} / \beta$ catenin signaling cascade. J Biol Chem. 2004;279:14879-88.

33 Möller S, Vilo J, Croning MD. Prediction of the coupling specificity of $\mathrm{G}$ protein coupled receptors to their $\mathrm{G}$ proteins. Bioinformatics. 2001;17:S174-181.

34 Koval A, Katanaev VL. Wnt3a stimulation elicits G-proteincoupled receptor properties of mammalian Frizzled proteins. Biochem J. 2011:433:435-40.

35 Kilander MBC, Petersen J, Andressen KW, Ganji RS, Levy FO, Schuster J, et al. Disheveled regulates precoupling of heterotrimeric G proteins to Frizzled 6. FASEB J. 2014;28:2293-305.

36 Dorsam RT, Gutkind JS. G-protein-coupled receptors and cancer. Nat Rev Cancer. 2007;7:79-94.

37. Mankin HJ, Dorfman H, Lippiello L, Zarins A. Biochemical and metabolic abnormalities in articular cartilage from osteo-arthritic human hips. J Bone Jt Surg Am. 1971;53:523-37. 